\title{
The effect of timing of teriparatide treatment on the circadian rhythm of bone turnover in postmenopausal osteoporosis
}

\author{
Maria Luchavova, Vit Zikan, Dana Michalska, Ivan Raska Jr, Ales A Kubena ${ }^{1}$ and Jan J Stepan ${ }^{2}$ \\ Department of Internal Medicine III-Department of Endocrinology and Metabolism, First Faculty of Medicine, Charles University in Prague and General \\ University Hospital in Prague, U Nemocnice 1, Cz-128 08 Prague, Czech Republic, ${ }^{1}$ Institute of Information Theory and Automation, Czech Academy of \\ Sciences, Cz-182 08 Prague, Czech Republic and ${ }^{2}$ Institute of Rheumatology, Charles University Faculty of Medicine 1, Cz-128 08 Prague, Czech Republic \\ (Correspondence should be addressed to V Zikan; Email: vit.zikan@lf1.cuni.cz)
}

\begin{abstract}
Background: We hypothesized that with the administration of teriparatide (TPTD) treatment at different times, we would be able to modify the physiological circadian rhythm of bone turnover.

Methods: The concentration of serum C-terminal telopeptide of collagen type I ( $\beta C T X)$, serum N-terminal propeptide of procollagen type I (P1NP), serum ionized calcium (iCa), and plasma PTH were measured every $3 \mathrm{~h}$ over a $24 \mathrm{~h}$ period in 14 postmenopausal osteoporotic women (aged 72.4 \pm 9.3 years) treated with $20 \mu \mathrm{g}$ TPTD for long term, given at different times of the day. General linear model-repeated measurements (GLM RM) were performed to analyze the circadian rhythms as well as intergroup comparisons.

Results: GLM-RM for both related groups showed a significant influence of time of day on all measured variables except P1NP. The analysis for each group separately provided a powerful model for $\beta$ CTX $\left(P<0.001, \eta^{2}=0.496\right)$, serum iCa $\left(P<0.001, \eta^{2}=0.423\right)$, plasma PTH $\left(P<0.001, \eta^{2}=0.283\right)$, and serum PINP $\left(P<0.001, \eta^{2}=0.248\right)$. While the evening TPTD treatment showed a marked circadian rhythm for serum $\beta C T X$, the morning TPTD treatment rather suggested circasemidian rhythm. The P1NP rhythm followed a much smaller amplitude of the rhythm than $\beta C T X$. Changes in serum iCa were positively related to changes in serum $\beta$ CTX $(P<0.001)$ and negatively related to changes in PTH $(P<0.001)$.

Conclusion: Timing of TPTD administration may significantly change the $24 \mathrm{~h}$ variation in bone turnover markers as well as calcium-parathyroid axis in postmenopausal osteoporotic women.
\end{abstract}

European Journal of Endocrinology 164 643-648

\section{Introduction}

Circadian rhythmicity is an essential feature of bone and mineral homeostasis. The biochemical markers of bone resorption, such as collagen type I degradation products, e.g. C-terminal or N-terminal telopeptide of type I collagen, follow a circadian rhythm, decreasing during the day and peaking at night $(1,2)$. For biochemical markers of bone formation, such as plasma osteocalcin and serum N-terminal or C-terminal propeptide of type I procollagen, a similar pattern of circadian rhythm with much smaller amplitude than that seen in bone resorption markers has been found (3-5). While rhythmicity of serum osteocalcin has been linked to cortisol secretion (4), the physiological basis for the circadian rhythm of bone resorption is not clearly understood and seems to be unaffected by age, menopausal status, gender, bed rest (2), or cortisol secretion (6). However, fasting significantly diminishes the circadian rhythm of bone-resorptive activity (7) and feeding deepens the morning decrease in bone-resorptive activity and accentuates its nocturnal peak (2). PTH is one of the most important systemic regulators of bone and mineral homeostasis, which follows a circadian rhythm and responds to feeding, especially to calcium/ phosphate intake. In healthy individuals, plasma concentrations of PTH show a circadian variability, with minimal concentrations around $1000 \mathrm{~h}$ and maximum levels at about $0300 \mathrm{~h}$ (8). Although the exact role for this rhythm has not been established yet, previous studies had showed an association between PTH rhythm and bone-resorptive activity (9). The PTH rhythm may set the amplitude of the circadian rhythm of bone resorption, especially by increasing the resorptive activity of existing osteoclasts during nocturnal fasting $(10,11)$. The nocturnal peak of PTH is abolished by fasting (12). The amplitude of the PTH circadian rhythm is smaller in osteoporotic women when compared with that seen in healthy women $(3,13,14)$. Studies in which calcium was administered for a longer time period either in the morning or in the evening, as opposed to acute suppression of PTH in clamp studies (11), have shown that timing of intermittent calcium supplementation influences the circadian rhythm of bone resorption (10). 
The aim of this study was to test our hypothesis that the effect of PTH treatment on bone remodeling and the calcium-PTH axis may be influenced by a different time of PTH administration. We investigated changes in bone turnover markers and the calcium-PTH axis over a $24 \mathrm{~h}$ period in postmenopausal osteoporotic women who were treated for a long term with teriparatide (TPTD), administered daily either in the morning or in the evening.

\section{Subjects and methods}

\section{Subjects}

All subjects were ambulatory women with established postmenopausal osteoporosis, 50-85 years of age, recruited from the Bone Center at the University Hospital, Prague, from July to December 2008. Patients were included if they were at least 5 years postmenopause, had a bone mineral density (BMD) T-score below -2.5 at the lumbar spine and/or the femoral neck, and had been treated with TPTD for at least 6 months, given either in the morning (approximately at $0800 \mathrm{~h}$, before breakfast) or in the evening (approximately at $0200 \mathrm{~h}$, after dinner). All patients had a total daily calcium intake of at least $1000 \mathrm{mg}$, either through diet or through diet enriched with calcium supplements, and were supplemented with vitamin D3 (800-1000 IU daily). Exclusion criteria were patients with abnormal serum or urinary calcium levels, with vitamin D insufficiency (serum 25-hydroxyvitamin D concentration $<50 \mathrm{nmol} / \mathrm{l}$ ); diseases other than osteoporosis intervening with bone metabolism; and treatment with other drugs affecting bone metabolism such as corticosteroids, cyclosporine, fluoride, or thiazide diuretics. Out of 20 invited patients, 14 women were eligible for the study. The study was attempted with the understanding and written consent of each subject, with the approval of the ethics committee of the Faculty of Medicine, Charles University, Prague, and in compliance with national legislation and the code of ethical principles for medical research involving human subjects of the World Medical Association (Declaration of Helsinki).

\section{Study design}

All subjects continued their TPTD and calcium and vitamin D supplements on the day before the study and fasted overnight before the start of the study. Standardized diet was served from 0800 to $0830 \mathrm{~h}, 1130$ to $1200 \mathrm{~h}$, and 1730 to $1800 \mathrm{~h}$. The intake of fluid was defined. The period of sleep and wakefulness was unbroken. Blood samples were obtained in the $3 \mathrm{~h}$ intervals via indwelling venous cannulae immediately before the administration of TPTD and meal intake $(0800 \mathrm{~h})$ in the fasted state and over the next $24 \mathrm{~h}$. TPTD was administrated either in the morning (0800 h) or in the evening $(2000 \mathrm{~h})$. The plasma and serum specimens were stored at $-70{ }^{\circ} \mathrm{C}$. Measurement of the serum ionized calcium (iCa) was performed on the same day after anaerobic collection of all specimens, which were anaerobically stored at $4{ }^{\circ} \mathrm{C}$ to avoid $\mathrm{pH}$ shifts.

\section{Biochemical analysis}

All samples, except the serum iCa, were simultaneously assayed. Serum iCa was measured using the ionselective electrode with an AVL 9180 (Roche Diagnostics $\mathrm{GmbH}$ ). The within-run imprecision was below $2 \%$ and between-run imprecision was below $4 \%$. The plasma concentrations of the immunoreactive intact PTH were determined using an electrochemiluminescence-based immunoanalysis (the Elecsys 1010 Analyzer, Roche Diagnostics GmbH). The withinrun imprecision was below $6 \%$.

The serum concentrations of $\beta$ CTX were measured using the electrochemiluminescence-based immunoanalysis (the Elecsys 1010 Analyzer, Roche Diagnostics). The within-run imprecision for the $\beta$ CTX was below $7 \%$. The serum concentrations of intact N-terminal propeptide of type I procollagen (PINP) were measured by RIA (Procollagen Intact PINP, Orion Diagnostica, Espoo, Finland). The within-run imprecision was below 5\%.

Serum concentrations of 25-hydroxyvitamin D (25$\mathrm{OHD})$ were determined by using enzymoimmunoassay (OCTEIA-25-Hydroxy Vitamin-D, Immunodiagnostic Systems Limited, Bolton, UK). Concentrations of 25-OHD above $50 \mathrm{nmol} / \mathrm{l}$ were considered normal.

\section{Statistical analysis}

The general linear model-repeated measured variant (GLM-RM) was used to assess the $24 \mathrm{~h}$ cyclic changes in the measured variables. The circadian rhythm was considered proven if the model revealed either the existence of a characteristic curve describing the daily rhythm similar for all patients (influence by time) or the existence of two curves for each group significantly demonstrating the similarity of the rhythm within each group, yet differences between the groups (influence of time by group interaction). In addition to testing the significance of levels observed depending on the selected variables (group, time, and group by time interaction), we used GLM RM to estimate the proportion of explained variability and thus effect size of this dependence $\left(\eta^{2}\right)$. Cohen's convention indicates a large size effect threshold $\eta^{2}>0.137$. GLM-RM also served for intergroup comparisons. With Pearson's correlation coefficient, calculated for the entire sample and for each group separately, we explored the relationship between changes in the levels of serum $\beta C T X$, iCa, and PTH during adjacent sampling (difference of $3 \mathrm{~h}$ each). The cosinor model $Y=$ mesor + AMP $\times \operatorname{COS}(2 \pi / 24$ $t+$ phase) has been used as an adjunct to the analysis of circadian rhythms. A description of the method and 
Table 1 Patients' characteristics at baseline. Values are mean \pm s.D. Differences between groups were analyzed by unpaired $t$-test.

\begin{tabular}{lccl}
\hline & $\begin{array}{c}\text { Morning } \\
\text { TPTD }\end{array}$ & $\begin{array}{c}\text { Evening } \\
\text { TPTD }\end{array}$ & \multicolumn{1}{c}{$\boldsymbol{P}$} \\
\hline Number of patients & 7 & 7 & - \\
Age (years) & $69.7 \pm 9.2$ & $75.1 \pm 9.1$ & $\mathrm{NS}$ \\
Weight $(\mathrm{kg})$ & $68.6 \pm 7.0$ & $53.9 \pm 4.1$ & 0.004 \\
Height $(\mathrm{m})$ & $1.65 \pm 0.06$ & $1.53 \pm 0.06$ & 0.006 \\
BMl $\left(\mathrm{kg} / \mathrm{m}^{2}\right)$ & $25.6 \pm 2.6$ & $23.0 \pm 1.1$ & $\mathrm{NS}$ \\
Lumbar spine BMD (T-score) & $-3.2 \pm 0.7$ & $-3.1 \pm 0.8$ & $\mathrm{NS}$ \\
Total hip BMD (T-score) & $-2.1 \pm 0.9$ & $-2.4 \pm 0.7$ & $\mathrm{NS}$ \\
Duration of TPTD treatment & $9.4 \pm 1.8$ & $11.9 \pm 2.3$ & $\mathrm{NS}$ \\
$\quad(\mathrm{months})$ & $1.29 \pm 0.05$ & $1.31 \pm 0.05$ & $\mathrm{NS}$ \\
S-iCa $(\mathrm{mmol} / \mathrm{l})$ & $28.9 \pm 7.4$ & $25.8 \pm 11.3$ & $\mathrm{NS}$ \\
P-PTH $(\mathrm{ng} / \mathrm{l})$ & $662.5 \pm 168$ & $940.1 \pm 461$ & $\mathrm{NS}$ \\
S- $\beta C T X(\mathrm{ng} / \mathrm{l})$ & $118.3 \pm 39$ & $169.9 \pm 92$ & $\mathrm{NS}$ \\
S-PINP $(\mu \mathrm{g} / \mathrm{l})$ & &
\end{tabular}

$\mathrm{BMI}$, body mass index; $\mathrm{BMD}$, bone mineral density; S-iCa, serum ionized calcium; P-PTH, plasma intact PTH; S- $\beta C T X$, serum C-terminal telopeptide of collagen type I; S-PINP, serum propeptide of procollagen type I.

its results is presented in the section titled 'Additional analysis of circadian rhythms'. All the statistical tests were performed with SPSS for Windows software, version 16.0 (SPSS Inc., Chicago, IL, USA).

\section{Results}

The patients' characteristics and the baseline values of the measured biochemical parameters are given in Table 1.

Analysis of the variations of serum $\beta$ CTX indicated a significant influence of time of day on the measured value (for time $P<0.001, \eta^{2}=0.492$, large size effect by Cohen's convention). These results suggest that there is a characteristic curve of $\beta C T X$ levels, which is inherent in all patient groups studied and which explains $49.2 \%$ of interindividual variability in measured levels of $\beta$ CTX. Further analysis showed a more powerful model, which characterizes the two curves, for each group separately (time versus group interaction, $P<0.001, \eta^{2}=0.496$ ). In the evening TPTD group, serum $\beta$ CTX levels indicated a circadian rhythm, whereas the morning TPD treatment group suggested a circasemidian rather than a circadian rhythm (Fig. 1A and B). The difference in $24 \mathrm{~h}$ mean $\beta C T X$ concentrations between treatment groups did not approach significance $(P=0.098)$; however, the power of the test was low (observed power $=0.379$ ). Analysis of the $12 \mathrm{~h}$ response curves showed a significantly greater daily mean value in serum $\beta$ CTX levels (between 2000 and $0800 \mathrm{~h}$ ) for the evening TPTD group compared with the morning TPTD group $\left(P=0.033, \eta^{2}=0.326\right.$; Fig. $1 \mathrm{~A}$ and $\left.\mathrm{B}\right)$.

For serum P1NP, GLM-RM did not prove the influence of time of day on the measured value for the related groups $(P=0.298)$. The analysis based on the two curves for each group separately demonstrated significantly different P1NP rhythms (time by group interaction, $\left.P<0.001, \eta^{2}=0.248\right)$, however, with much smaller amplitude than the bone resorption marker (Fig. 2A and B; Table 2). Probably, due to the wide inter-individual variability, the differences in $24 \mathrm{~h}$ mean values between morning and evening TPTD administration could not be detected.

Analysis of variations of iCa indicated a significant time effect $(P=0.021)$, grouped by time interaction $(P<0.001)$ and a large size effect by Cohen's convention $\left(\eta^{2}=0.180\right.$ and $\eta^{2}=0.423$, time and group by time interaction respectively). Curves from both groups were running against each other (i.e. high levels for the morning group occur at times of low levels for the evening group and vice versa (Fig. $3 \mathrm{~A}$ and $\mathrm{B})$ ) and the common curve explained $18.0 \%$ of inter-individual variability in measured levels of serum iCa. The analysis after distribution explained $42.3 \%$ of the variability. The difference in $24 \mathrm{~h}$ mean iCa concentrations between treatment groups did not approach significance $(P=0.973)$; however, the power of the tests is extremely low (0.050). Also, analysis of the $12 \mathrm{~h}$ response curves showed no significant difference between treatment groups, probably due to high
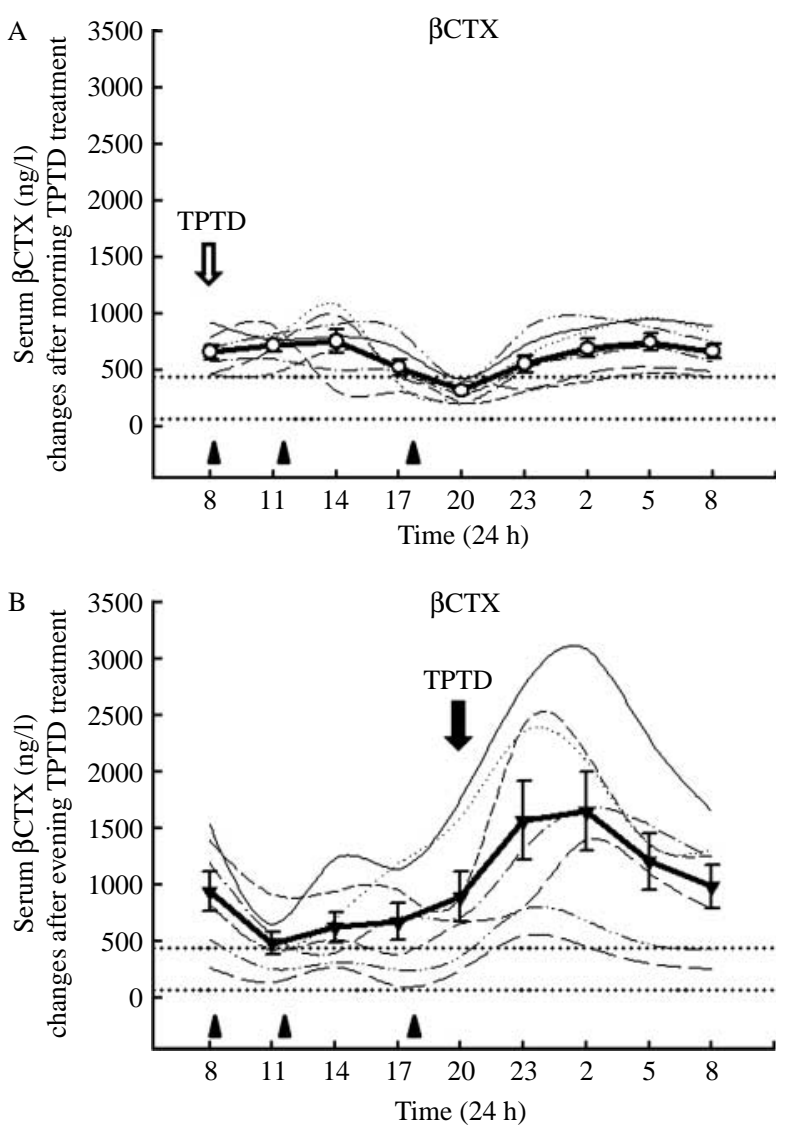

Figure 1 ( $A$ and $B$ ) Changes in serum $\mathrm{C}$-terminal telopeptide of collagen type I ( $\beta C T X)$ either after morning $(A)$ or after evening $(B)$ teriparatide (TPTD) treatment. Each hairline represents one individual. The bold line represents the mean \pm S.E.M. Dotted lines represent normal reference range. TPTD administration is indicated by bold arrow. Meals were taken at the times indicated by triangles (0800-0830 h; 1130-1200 h; and 1730-1800 h). 

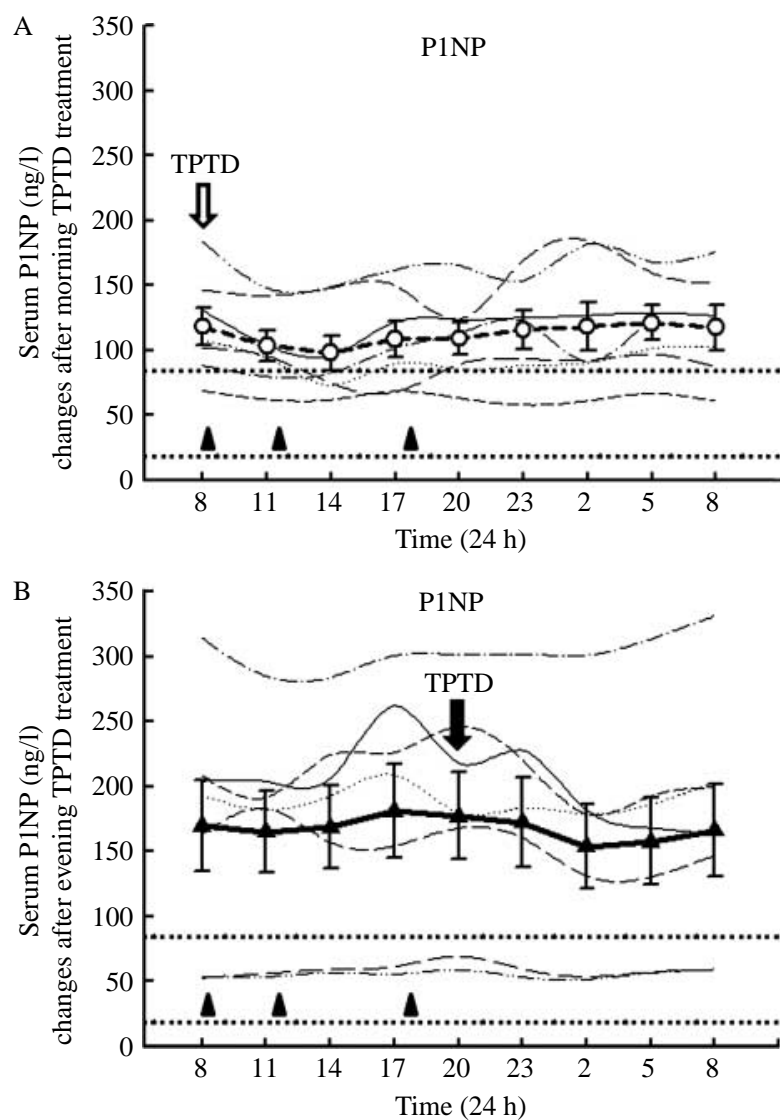

Figure 2 ( $A$ and $B$ ) Changes in serum $\mathrm{N}$-terminal propeptide of procollagen type I (P1NP) either after morning $(A)$ or after evening (B) teriparatide (TPTD) treatment. Each hairline represents one individual. The bold line represents the mean \pm S.E.M. Dotted lines represent normal reference range. TPTD administration is indicated by bold arrow. Meals were taken at the times indicated by triangles (0800-0830 h; 1130-1200 h; and 1730-1800 h).

interindividual variability. However, serum iCa levels were positively related to bone-resorptive activity as assessed by serum $\beta$ CTX levels $(P<0.001)$.

Analysis of variations of endogenous plasma PTH showed a significant time effect $(P=0.038)$, and time by group interaction $(P<0.001)$, and large size effect by Cohen's convention (time and time by group interaction, $\eta^{2}=0.152$ and $\eta^{2}=0.283$ respectively). Endogenous PTH mirrored, in the opposite direction, the changes in serum iCa (Fig. 3A and B). A significant negative correlation was found between iCa and PTH changes $(P<0.001)$. Daily mean PTH levels were not different between the groups $(P=0.572)$, but the power of the test was extremely low (0.083).

\section{Additional analysis of circadian rhythms}

The cosinor model $Y=$ mesor $+\mathrm{AMP} \times \operatorname{COS}(2 \pi / 24$ $t+$ phase) has been used as an adjunct to the analysis of circadian rhythms. We used this model with a restriction for certain estimates. One of the assumptions of the cosinor model, in sharp contrast with most of our measured data, is a requirement that the daily maximal and daily minimal values are occurred in the opposition, i.e. $12 \mathrm{~h}$ after each other. Therefore, the estimates of the phase, estimated time for maximum, and estimated time for the minimum cannot be applied to our data, contrary to the estimates of the MESOR and amplitude has no effect. The results of the cosinor model are shown in Table 2. Out of the variables, only the amplitude of S- $\beta$ CTX after evening administration of TPTD was significantly greater $(P<0.05)$.

\section{Discussion}

It is well known that treatment with exogenous PTH exerts both anabolic and catabolic effects on bone, depending on the concentration and duration of exposure (15); however, because of the physiological circadian rhythms of bone turnover and PTH, there is a possibility that the effect of TPTD treatment on bone turnover may also be influenced by the time of TPTD administration during the day. Despite the great interest in PTH as a therapeutic agent, the effect of timedependent PTH treatment on bone turnover has not been studied previously.

In this study, we found that time-dependent TPTD treatment is able to significantly change the $24 \mathrm{~h}$ variation in bone turnover markers, as well as serum calcium and endogenous PTH secretion, in women with established postmenopausal osteoporosis. Whereas the evening TPTD treatment $(2000 \mathrm{~h})$ followed the circadian rhythm of bone resorption marker $\beta$ CTX with a nadir between 1100 and $1400 \mathrm{~h}$ and a peak between 2300 and $0200 \mathrm{~h}$, the morning TPTD treatment $(0800 \mathrm{~h})$ resulted in an increase in BCTX between 0800 and $1400 \mathrm{~h}$ and a subsequent decrease until $2000 \mathrm{~h}$, which was followed by a significant increase in bone resorption marker, suggesting rather

Table 2 Circadian rhythm analysis (cosinor model).

\begin{tabular}{lllcc}
\hline & MESOR & Amplitude & $\begin{array}{c}\text { Max } \\
\text { (estimate) } \\
(\mathrm{h})\end{array}$ & $\begin{array}{c}\text { Min } \\
\text { (estimate) } \\
\text { (h) }\end{array}$ \\
\hline S- $\beta$ CTX_morning & 585,7 & 157,8 & 0748 & 1948 \\
S- $\beta$ CTX_evening & 990,9 & $522,7^{\star}$ & 0118 & 1318 \\
S-iCa_morning & 1,3 & 0,02 & 1336 & 0136 \\
S-iCa_evening & 1,3 & 0,04 & 0248 & 1448 \\
S-PINP_morning & 111,9 & 10,5 & 0300 & 1500 \\
S-PINP_evening & 168,3 & 9,1 & 1700 & 0500 \\
P-PTH_morning & 22,6 & 2,8 & 0236 & 1436 \\
P-PTH_evening & 25,2 & 2,9 & 1512 & 0312 \\
\hline
\end{tabular}

${ }^{*} P<0.05, \mathrm{~S}-\beta \mathrm{CTX}$ evening versus morning amplitude; all statistical tests were performed with SPSS for Windows software, version 16.0. MESOR midline estimate statistic of rhythm; S-iCa $(\mathrm{mmol} / \mathrm{l})$, serum ionized calcium; P-PTH (ng/l), plasma intact PTH; S- $\beta$ CTX (ng/l), serum C-terminal telopeptide of collagen type I; S-PINP $(\mu \mathrm{g} / \mathrm{l})$, serum propeptide of procollagen type I; Max (estimate), estimation time of daily maximum (h); Min (estimate), estimation time of daily minimum $(\mathrm{h})$ 

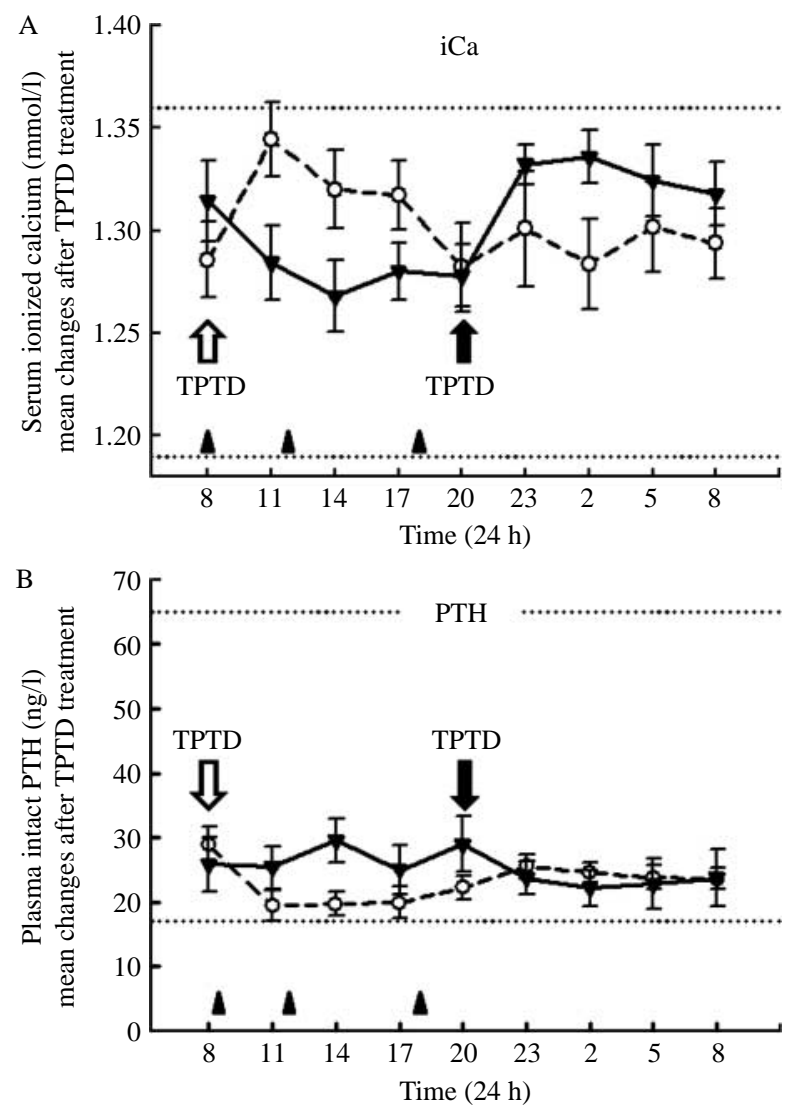

Figure 3 ( $A$ and $B$ ) Mean changes \pm S.E.M. in serum ionized calcium ( $\mathrm{iCa}$ ) and plasma intact parathyroid hormone $(\mathrm{PTH})$ either after morning (A) or after evening (B) teriparatide (TPTD) treatment. Dotted lines represent normal reference range. TPTD administration is indicated by bold arrow. Meals were taken at the times indicated by triangles (0800-0830 h; 1130-1200 h; and 1730-1800 h).

circasemidian than circadian rhythm. The concentrations of serum iCa transiently but significantly increased 3-6 h after TPTD administration in both treatment groups (Fig. 3A). Parallel movement of serum $\mathrm{iCa}$ and $\beta \mathrm{CTX}$, which were significantly related to each other, indicates that changes in serum iCa reflect changes in osteoclast-resorptive activity. The PTH serum concentrations significantly decreased $3-6 \mathrm{~h}$ after TPTD administration, and the profiles of serum iCa and endogenous PTH were significantly inversely related and mirrored with each other, indicating that the parathyroid function is preserved during TPTD treatment. These results are in accordance with the previous studies in which an inverse correlation between circadian changes in serum iCa and endogenous PTH has been demonstrated $(16,17)$.

Our results indicate that the sensitivity of osteoclasts to TPTD treatment varies by the time of TPTD dosing. The percentage increase in serum $\beta$ CTX during the first $6 \mathrm{~h}$ after TPTD administration was significantly more pronounced after the evening TPTD administration in comparison with its morning administration
$(P=0.009 ; 22 \pm 56$ and $94 \pm 38 \%$; morning and evening TPTD treatment respectively). The mechanism(s) of this phenomenon is presently uncertain; however, the results imply a homeostatic mechanism that limits response of bone resorption to TPTD treatment in the morning and/or augments its response in the evening. Although we did not investigate the mechanisms underlying this phenomenon, we suggest at least two factors that may modulate the bone remodeling balance during the TPTD treatment. First, at a cellular level, the increase in bone-resorptive activity by PTH is believed to be an indirect effect of PTH via the production of local factors, such as RANKL from osteoblasts and/or a transient decrease in the production of osteoprotegerin $(\mathrm{OPG})$, which acts as a decoy receptor for RANKL $(18,19)$. A recent study by Joseph et al. (9) demonstrated a circadian rhythm for circulating OPG levels with a daytime increase and nocturnal decrease in healthy subjects. Therefore, the endogenous circadian rhythm in OPG secretion may at least partly modulate the effect of TPTD treatment on osteoclast's resorptive activity. However, further studies are needed to test this possibility in postmenopausal osteoporotic women. Secondly, the effect of PTH injection on osteoclasts may be attenuated due to the known suppressive effect of food intake on boneresorptive activity in the morning (20). Interestingly, osteoclasts are less affected by feeding in the late afternoon and evening in non-fasting individuals (21). Further investigation is required for better understanding of these complex regulations and to optimize bone remodeling balance during TPTD treatment in postmenopausal women with osteoporosis.

In this study, serum PINP significantly varied according to the time of TPTD administration $(P=0.001)$ but to a much smaller extent than the bone resorption marker. Serum PINP showed a mild but significant decrease during $6 \mathrm{~h}$ after the morning $(P=0.033)$ or evening $(P<0.05)$ TPTD administration, with a subsequent mild increase in both treatment groups. The delayed response of serum PINP in our study may suggest that collagen synthesis requires more time than collagen degradation to show acute changes after TPTD administration. The inter-individual mean levels in PINP were highly variable and the overall mean $24 \mathrm{~h}$ level of PINP was not significantly different between the groups.

Several limitations of this study must be taken into account. First, due to the cross-sectional design, we could not evaluate the changes in $24 \mathrm{~h}$ variation in the measured parameters before and after TPTD treatment. Secondly, due to the small number of subjects and a high inter-individual variability, some differences did not reach statistical significance. However, our results clearly showed that the time of TPTD administration has a large effect on the measured variables. Thirdly, a more frequent sampling would be 
required to study the $24 \mathrm{~h}$ variation of serum iCa and plasma endogenous PTH (9).

In conclusion, this is the first study to show that circadian variation in bone-resorptive activity significantly differs according to the dosing time of the TPTD treatment. Our data support our hypothesis that the response of bone cells to TPTD treatment differs by its dosing time. Whether the timing of TPTD administration will have an impact on the efficacy and safety of long-term TPTD treatment will need to be further analyzed in prospective studies. With respect to longterm efficacy, several parameters such as BMD need to be evaluated.

\section{Declaration of interest}

The authors declare that there is no conflict of interest that could be perceived as prejudicing the impartiality of the research reported.

\section{Funding}

This work was supported by IGA Ministry of Health of Czech Republic NS 10564-3.

\section{Acknowledgement}

We thank Oldriska Lukaskova for excellent technical assistance.

\section{References}

1 Wichers M, Schmidt E, Bidlingmaier F \& Klingmüller D. Diurnal rhythm of CrossLaps in human serum. Clinical Chemistry 199945 1858-1860.

2 Qvist P, Christgau S, Pedersen BJ, Schlemmer A \& Christiansen C. Circadian variation in the serum concentration of C-terminal telopeptide of type I collagen (serum CTx): effects of gender, age, menopausal status, posture, daylight, serum cortisol, and fasting. Bone 200231 57-61. (doi:10.1016/S8756-3282(02)00791-3)

3 Eastell R, Calvo MS, Burritt MF, Offord KP, Russell RG \& Riggs BL. Abnormalities in circadian patterns of bone resorption and renal calcium conservation in type I osteoporosis. Journal of Clinical Endocrinology and Metabolism 199274 487-494. (doi:10.1210/ jc.74.3.487)

4 Nielsen HK, Brixen K, Kassem M, Charles P \& Mosekilde L. Inhibition of the morning cortisol peak abolishes the expected morning decrease in serum osteocalcin in normal males: evidence of a controlling effect of serum cortisol on the circadian rhythm in serum osteocalcin. Journal of Clinical Endocrinology and Metabolism 199274 1410-1414. (doi:10.1210/jc.74.6.1410)

5 Ahmad AM, Hopkins MT, Fraser WD, Ooi CG, Durham BH \& Vora JP. Parathyroid hormone secretory pattern, circulating activity, and effect on bone turnover in adult growth hormone deficiency. Bone 200332 170-179. (doi:10.1016/S8756-3282(02)00952-3)

6 Schlemmer A, Hassager C, Alexandersen P, Fledelius C, Pedersen BJ, Kristensen IO \& Christiansen C. Circadian variation in bone resorption is not related to serum cortisol. Bone 199721 83-88. (doi:10.1016/S8756-3282(97)00039-2)

7 Schlemmer A, Ravn P, Hassager C \& Christiansen C. Morning or evening administration of nasal calcitonin? Effects on biochemical markers of bone turnover Bone 199720 63-67. (doi:10.1016/ S8756-3282(96)00307-9)

8 el-Hajj Fuleihan G, Klerman EB, Brown EN, Choe Y, Brown EM \& Czeisler CA. The parathyroid hormone circadian rhythm is truly endogenous - a general clinical research center study. Journal of Clinical Endocrinology and Metabolism 199782 281-286. (doi:10.1210/jc.82.1.281)

9 Joseph F, Chan BY, Durham BH, Ahmad AH, Vinjamin S, Gallagher JA, Vora JP \& Fraser WD. The circadian rhythm of osteoprotegerin and its association with parathyroid hormone secretion. Journal of Clinical Endocrinology and Metabolism 200792 3230-3238. (doi:10.1210/jc.2007-0812)

10 Blumsohn A, Herrington K, Hannon RA, Shao P, Eyre DR \& Eastell R. The effect of calcium supplementation on the circadian rhythm of bone resorption. Journal of Clinical Endocrinology and Metabolism 199479 730-735. (doi:10.1210/jc.79.3.730)

11 Ledger GA, Burritt MF, Kao PC, O’Fallon WM, Riggs BL \& Khosla S. Role of parathyroid hormone in mediating nocturnal and age-related increases in bone resorption. Journal of Clinical Endocrinology and Metabolism 199580 3304-3310. (doi:10. $1210 /$ jc.80.11.3304)

12 Fraser WD, Logue FC, Christie JP, Cameron DA, O'Reilly DS \& Beastall GH. Alteration of the circadian rhythm of intact parathyroid hormone following a 96-hour fast. Clinical Endocrinology 199440 523-528. (doi:10.1111/j.1365-2265. 1994.tb02493.x)

13 Fraser WD, Logue FC, Christie JP, Gallacher SJ, Cameron D, O'Reilly DS, Beastall GH \& Boyle IT. Alteration of the circadian rhythm of intact parathyroid hormone and serum phosphate in women with established postmenopausal osteoporosis. Osteoporosis International 19988 121-126. (doi:10.1007/BF02672507)

14 Ledger GA, Burritt MF, Kao PC, O'Fallon WM, Riggs BL \& Khosla S. Abnormalities of parathyroid hormone secretion in elderly women that are reversible by short term therapy with 1,25-dihydroxyvitamin D3. Journal of Clinical Endocrinology and Metabolism 1994 79 211-216. (doi:10.1210/jc.79.1.211)

15 Dobnig H \& Turner RT. The effects of programmed administration of human parathyroid hormone fragment (1-34) on bone histomorphometry and serum chemistry in rats. Endocrinology 1997138 4607-4612. (doi:10.1210/en.138.11.4607)

16 Calvo MS, Eastell R, Offord KP, Bergstralh EJ \& Burritt MF. Circadian variation in ionized calcium and intact parathyroid hormone: evidence for sex differences in calcium homeostasis. Journal of Clinical Endocrinology and Metabolism 199172 69-76. (doi:10.1210/jcem-72-1-69)

17 Nielsen HK, Brixen K, Kassem M, Christensen SE \& Mosekilde L. Diurnal rhythm in serum osteocalcin: relation with sleep, growth hormone, and PTH(1-84). Calcified Tissue International 199149 373-377. (doi:10.1007/BF02555845)

18 Onyia JE, Miles RR, Yang X, Halladay DL, Hale J, Glasebrook A, McClure D, Seno G, Churgay L, Chandrasekhar S \& Martin TJ. In vivo demonstration that human parathyroid hormone 1-38 inhibits the expression of osteoprotegerin in bone with the kinetics of an immediate early gene. Journal of Bone and Mineral Research 200015 863-871. (doi:10.1359/jbmr.2000.15.5.863)

19 Ma YL, Cain RL, Halladay DL, Yang X, Zeng Q, Miles RR, Chandrasekhar S \& Martin TJ\& Onyia JE. Catabolic effects of continuous human PTH (1-38) in vivo is associated with sustained stimulation of RANKL and inhibition of osteoprotegerin and geneassociated bone formation. Endocrinology 2001142 4047-4054. (doi:10.1210/en.142.9.4047)

20 Clowes JA, Hannon RA, Yap TS, Hoyle NR, Blumsohn A \& Eastell R. Effect of feeding on bone turnover markers and its impact on biological variability of measurements. Bone $2002 \mathbf{3 0}$ 886-890. (doi:10.1016/S8756-3282(02)00728-7)

21 Bjarnason NH, Henriksen EE, Alexandersen P, Christgau S, Henriksen DB \& Christiansen C. Mechanism of circadian variation in bone resorption. Bone 200230 307-313. (doi:10.1016/ S8756-3282(01)00662-7)

Received 28 January 2011

Accepted 2 February 2011 